\title{
Théorie de l'imagination en France à l'aube des Lumières : Malebranche et Fontenelle
}

\begin{abstract}
RÉsUMÉ. - Cet article s'efforce de prendre la mesure de l'impact du cartésianisme dans les transformations du concept d'imagination à la fin du XVII siècle en prenant pour points d'ancrage les développements consacrés à cette notion chez Malebranche et Fontenelle. Ces deux penseurs accentuent des polarités qui sont présentes dans la conception cartésienne de l'imagination: Malebranche fait fond sur la notion d'imagination passive pour en faire le pivot d'une théorie de la contagion des affects et des représentations, alors que Fontenelle mobilise celle d'imagination active pour élaborer son histoire de l'esprit humain. Tous deux, à terme, font de l'imagination une notion centrale dans la formation d'un proto-concept de «culture».
\end{abstract}

ABSTRACT. - This article focuses on the impact of Cartesianism in the transformations of the concept of imagination at the end of the $17^{\text {th }}$ century, giving attention primarily to the thoughts of Malebranche and Fontenelle. These two thinkers emphasize polarities that are already present in the Cartesian conception of imagination: Malebranche uses the notion of passive imagination to theorize the contagion of affects and ideas, while Fontenelle elaborates his history of the human mind around the notion of active imagination. Both of them, in the end, give imagination a central role in the formation of a proto-concept of «culture».

Les métamorphoses et variations qui affectent le concept d'imagination au cours des $\mathrm{XVII}^{\mathrm{e}}$ et $\mathrm{XVIII}{ }^{\mathrm{e}}$ siècles constituent, à n'en pas douter, un symptôme particulièrement éclairant des transformations qui s'opèrent dans le champ global de la philosophie moderne. De fait, l'imagination voit sa sphère d'application s'étendre jusqu'à la fin du XVIII ${ }^{\mathrm{e}}$ siècle, au fur et à mesure des redéfinitions dont elle fait l'objet. Faculté de l'âme à la frontière du corps et de l'esprit, elle intervient dans ce qu'on nommerait aujourd'hui la philosophie de l'esprit tout autant que dans la théorie des passions, affectant ainsi de ses mutations la théorie de la connaissance, la philosophie morale, l'esthétique philosophique, et bien d'autres domaines. Tantôt vouée aux gémonies comme cause des plus graves erreurs dans lesquelles elle entraîne l'esprit, tantôt glorifiée pour sa fécondité, l'imagination, plus encore qu'un simple concept, constitue un véri- 
table nœud théorique où s'incarnent les tensions de la pensée moderne, résultats d'un amalgame de survivances du passé et d'efforts pour s'en défaire.

Si le constat n'est plus à faire de ce que l'imagination acquiert dans la seconde moitié $\mathrm{du} \mathrm{XVIII}{ }^{\mathrm{e}}$ siècle un statut théorique de première importance - qu'on pense à Hume, à Rousseau ou à Kant -, l'histoire de ce qui a rendu possible une telle promotion demeure fragmentaire, et ce particulièrement en ce qui a trait à la spécificité des Lumières françaises. L'évolution de la notion est généralement dessinée en prenant pour axe principal le développement de l'empirisme britannique ${ }^{1}$. Or, si de nombreux travaux récents se sont attachés à relever d'autres filiations qui mènent à la pensée des Lumières françaises ${ }^{2}$, peu d'entre eux se sont directement penchés sur le rôle de ces filiations dans l'évolution du concept d'imagination. Il semble pourtant qu'une telle enquête puisse s'avérer fertile pour comprendre certains traits communs aux philosophes des Lumières françaises qui les distinguent de leurs contemporains britanniques, allemands, italiens, etc., et ce qui divise ces mêmes philosophes entre divers réseaux d'appartenance théorique; de plus, une telle enquête permettrait de mieux saisir l'inflexion particulière que le concept d'imagination a prise durant cette période, et son impact sur les périodes subséquentes.

Loin de prétendre à l'exhaustivité d'une telle enquête, ce qui suit vise plutôt à en poser un certain nombre de jalons, délimitant ainsi quelques-uns des lieux théoriques autour desquels la question de l'imagination évolue tout au long du XVIII ${ }^{\mathrm{e}}$ siècle. De façon plus précise, il s'agit de dresser deux configurations conceptuelles - incarnées par Malebranche et Fontenelle - qui illustrent de manière exemplaire les déplacements qui affectent le concept d'imagination au croisement des XVII ${ }^{\mathrm{e}}$ et $\mathrm{XVIII}{ }^{\mathrm{e}}$ siècles, déplacements qui anticipent sur l'avenir de

1. Voir par exemple la magistrale étude d'Annie BECQ, qui consacre de nombreux chapitres à la question de l'imagination, Genèse de l'esthétique française moderne, Paris, Albin Michel, 1994, pp. $145 \mathrm{sq}$. L'auteure, cependant, ne limite pas son analyse à montrer l'apport de l'empirisme à la théorie de l'imagination, puisque dans les chapitres précédents des développements sont consacrés à l'héritage cartésien et gassendien. Seulement dans les pages citées, elle affirme que la promotion de l'imagination en France est un effet de l'accroissement de popularité de l'empirisme.

2. Nous pensons ici aux travaux récents d'horizons divers qui ont permis de faire remonter à la surface les réseaux clandestins précurseurs des Lumières militantes de la seconde moitié du XVIII ${ }^{\mathrm{e}}$ siècle, comme, par exemple, Richard Henry PopkIN, Ezequiel DE Olaso et Giorgio TonelLI, Scepticism in the Enlightenment, Dordrecht, Kluwer Academic, 1997; Jonathan I. IsRAEL, Radical Enlightenment. Philosophy and the Making of Modernity 1650-1750, New York, Oxford University Press, 2001. À cela, il faut joindre les travaux actuels qui s'attachent à montrer la persistance de motifs cartésiens passés au crible malebranchiste ou leibnizien, parmi lesquels André CHARRAK, Contingence et nécessité des lois de la nature au XVIII siècle: la philosophie seconde des Lumières, Paris, Vrin, 2006; Delphine Kolesnik-Antoine, L'Homme cartésien. La "force qu'a l'âme de mouvoir le corps », Rennes, Presses universitaires de Rennes, 2009. Parmi les études plus datées qui faisaient déjà une place à ce type de filiations, on notera J. H. BRUMFITT, The French Enlightenment, Cambridge, Shenkman Publishing, 1972; Ira O. WAdE, The Intellectual Origins of the French Enlightenment, Princeton, Princeton University Press, 1971. 
la notion, ou en constituent les conditions historiques de possibilité. Par configuration conceptuelle, il faut entendre ici un réseau de concepts qui permet de saisir une notion avec ses différentes fonctions dans un système d'énoncés, les phénomènes qui y sont associés et l'explicitation de son fonctionnement. Ainsi, eu égard à l'imagination, parler de configuration conceptuelle signifie déterminer, au-delà de sa seule définition, le rapport qu'elle entretient avec les autres facultés de l'âme, la nature et les effets de ses manifestations et le rôle qui lui est imputé dans l'explication de divers phénomènes.

Le choix de Malebranche et de Fontenelle, dans ce contexte, n'est pas fortuit. Il découle de trois considérations principales:

1) D'abord, tous deux incarnent, chacun à sa manière, un type de prolongement du cartésianisme ${ }^{3}$. Ainsi, si Malebranche, plus préoccupé de développer les conséquences métaphysiques du dualisme des substances posé par Descartes, a proposé de faire le système des causes occasionnelles pour rendre compte de la nécessité à l'œuvre dans la nature, Fontenelle, pour sa part, requalifiant la mathesis universalis en l'incorporant dans un cadre expérimentaliste, s'est efforcé de propager la science cartésienne en laissant de côté son cadre métaphysique de référence, et ce jusque dans la seconde moitié du XVIII ${ }^{\mathrm{e}}$ siècle, alors que le newtonianisme s'est imposé de façon quasi générale. Cet ancrage commun dans le cartésianisme de deux auteurs si différents met au jour le fait que la conceptualité cartésienne pouvait donner prise à des interprétations difficilement compatibles ${ }^{4}$. Il faudra se demander, en ce qui a trait à l'imagination, dans quelle mesure ces valences appartiennent à la pensée de Descartes, ou si l'écart qui se creuse à l'intérieur de son héritage est tributaire d'une redéfinition de l'imagination par l'un ou l'autre des partis en présence.

2) Une seconde considération dérive de la postérité de ces deux auteurs dans les Lumières françaises. Bien que très diversifiée, la réception de ces deux œuvres par les penseurs français de la seconde moitié du XVIII ${ }^{\mathrm{e}}$ siècle montre qu'il s'agit de références communes. Malebranche et Fontenelle constituent en ce sens des opérateurs historiques importants pour comprendre la façon dont les problèmes posés par la philosophie cartésienne se présentent à la génération qui

3. J. H. Brumfitt (op. cit.) parle, pour Fontenelle, d'un cartésianisme scientifique, intégrant ses positions mécanistes dans un ensemble plus près du matérialisme gassendiste, qu'il oppose au cartésianisme épistémologique de Malebranche, qui met plutôt l'accent sur ses présupposés métaphysiques.

4. On notera d'ailleurs qu'il y a eu confrontation entre ces deux avatars du cartésianisme en 1686, alors que Fontenelle publie ses Doutes sur le système physique des causes occasionnelles, qui donnent lieu à un échange avec Bayle, puis à des Réflexions sur les "Doutes », vraisemblablement dues à Malebranche lui-même. Sur cette question, voir FonTEnelle, Euvres complètes, Paris, Fayard, 1990-1997, vol. I, pp. 6, 525-582. Désormais, nous ferons toujours référence à cette édition suivant la forme: $O C,\left[\mathrm{n}^{\circ}\right.$ de volume], [n ${ }^{\circ}$ de page]. 
leur succède. En ce qui a trait spécifiquement à la question de l'imagination, on trouve un exemple frappant de la façon dont la pensée de Malebranche devient un objet de débat dans l'article Imagination de l'Encyclopédie ${ }^{5}$. La première partie de l'article, qui est de la main de Voltaire, affirme que cette faculté est responsable du passage «dans les enfants [des] marques évidentes d'une impression qu'une mère a reçue ${ }^{6}$. Or, la seconde partie de l'article, intitulée «Imagination des femmes enceintes sur le fotus, pouvoir de $l^{\prime} »^{7}$, fournit un long développement sur cette théorie en en faisant remonter l'origine à Malebranche, développement qui commence par une remarque assassine, affirmant que ces effets,

s'ils dépendent de l'imagination, doivent bien plus raisonnablement être attribués à celle des personnes qui croient les apercevoir, qu'à celle de la mère, qui n'a réellement, ni n'est susceptible d'avoir aucun pouvoir de cette espèce ${ }^{8}$.

Cette présence d'une confrontation ouverte dans l'Encyclopédie, procédé récurrent utilisé par Diderot lorsqu'il veut mettre en cause les propos d'un article qu'il a commandé, suffit à illustrer que les thèses de Malebranche sur la transmission des «traces » de la mère à son fœtus - sur lesquelles on reviendra plus loin - forment un lieu théorique associé à la théorie de l'imagination sur lequel il est désormais nécessaire de prendre position.

3) Enfin, d'un point de vue plus philosophique, la mise en regard des thèses de Fontenelle et de Malebranche apparaît propre à relever, au-delà des tensions qu'elles recouvrent, les lignes de convergence autour desquelles l'unité de la notion se structure tout en lui donnant une configuration particulière. De manière très schématique, on peut déjà dire que cette unité renvoie, à l'intérieur du cadre général de ce qu'on peut appeler la critique de la superstition, à l'analyse des phénomènes qui conduisent la formation de l'esprit et des mours d'une nation. L'imagination est en effet associée par ces deux auteurs à la dynamique responsable de la formation de représentations imaginaires et aux mécanismes de leur transmission. En ce sens, l'imagination jouera un rôle crucial dans l'éta-

5. L'influence globale de Malebranche sur la théorie de l'imagination au XVIII ${ }^{\mathrm{e}}$ siècle a été étudiée dans J. MARX, «Le concept d'imagination au XVIII ${ }^{\mathrm{e}}$ siècle», in Raymond Trousson (dir.), Thèmes et figures des Lumières. Mélanges offerts à Roland Mortier, Genève, Droz, 1980, pp. 147-160.

6. Encyclopédie ou Dictionnaire raisonné des sciences, des arts et des métiers, par une Société de Gens de lettres, Paris, Briasson, 1751-1765, vol. VIII, p. 561 [orthographe modernisée]. Pour l'attribution de l'article, voir l'introduction de VoltaIRE, Euvres complètes de Voltaire, Oxford, Voltaire Foundation, vol. 33 (1) - Euvres alphabétiques, J. Vercruysse (dir.).

7. Cet article est attribué à «Diderot3 » par le projet ARTFL, marque qui désigne les articles non signés qu'on peut raisonnablement, mais de manière encore incertaine, attribuer à Diderot. Sur cette question, voir: http://encyclopedie.uchicago.edu.proxy.bib.uottawa.ca/node/163.

8. Encyclopédie, io. cit., VIII, p. 563 [orthographe modernisée]. 
blissement d'une véritable conception historique de la science, des arts et des mœurs.

C'est en gardant ces trois considérations en vue que les définitions malebranchiste et fontenellienne de l'imagination seront traitées dans ce qui suit. Un traitement séparé de ces définitions s'imposait de par la nature même des corpus au sein desquels elles se présentent. Le caractère systématique de l'œuvre principale de Malebranche, De la recherche de la vérité, permettait en effet de concentrer l'attention sur la version définitive du chapitre entièrement consacré à l'imagination (livre II), qui fournit l'essentiel des fondations théoriques qui régissent l'emploi du concept dans le reste de l'œuvre - tout particulièrement au livre V, qui porte sur les passions. En revanche, l'œuvre de Fontenelle commandait une reconstruction de la notion à partir de considérations parsemées dans des ouvrages de nature très diverse. Les textes écrits dans la période qui précède son entrée à l'Académie (1700), et particulièrement ceux concernant l'origine des «fables », ont été privilégiés parce qu'ils contiennent des indications décisives sur sa conception de l'imagination.

\section{DESCARTES. PASSIVITÉ ET ACTIVITÉ DE L'IMAGINATION}

L'imagination, chez Descartes, même si elle désigne une faculté de produire des représentations sensibles, n'est pas, au sens propre, une force. Elle est une matière. Sa définition bivalente en termes d'imagination passive et d'imagination active $^{9}$ a permis de montrer que les images qui s'y forment résultent soit du mouvement des esprits animaux (lui-même soit fortuit, soit causé par les corps extérieurs), soit de la force conjuguée de la volonté et de l'entendement. Cette distinction fondamentale, c'est elle qui, en quelque sorte, régule l'ensemble du discours cartésien sur l'usage de l'imagination.

Des Regulae aux Principia, l'accent est mis, dans les écrits sur la connaissance, sur l'imagination active, c'est-à-dire sur son emploi procédural. De la réduction des objets de la connaissance à des grandeurs représentées par des figures géométriques à la formation de l'algèbre (Règles 14 à 16), les Regulae montrent comment l'entendement peut recourir à des objets à deux dimensions qu'il crée de toutes pièces dans le matériau de l'imagination pour s'aider à voir et à se rappeler les opérations qu'il fait et leurs résultats. Dans les Principia, l'imagination est mise au service de la construction d'entités théoriques, comme les tourbillons de fluide qui entraînent les planètes dans leur mouvement : l'ima-

9. Les Passions de l'âme, $\S \S 19-21$, in Euvres de Descartes, édition de Ch. Adam et P. Tannery, Paris, Cerf, 1897-1909, XI, pp. 343-345. 
gination est ainsi mise à profit pour la connaissance des choses matérielles par le moyen de leur reconstitution progressive à partir des lois de la géométrie et de l'arithmétique maîtrisées par l'entendement ${ }^{10}$.

L'imagination active est, sous ce rapport, un pouvoir de conception. Mais, comme les Méditations l'ont montré, il s'agit d'un pouvoir de conception limité par sa dimension sensible. L'exemple du morceau de cire de la Seconde Méditation a servi à montrer que la connaissance par imagination est, comme la connaissance sensible, inapte à saisir l'essence de l'objet qu'elle considère ${ }^{11}$. Lorsqu'elle se donne l'image d'un objet, elle n'accède pas à son concept, mais à l'une de ses manifestations possibles. C'est là sa limite ontologique : en tant que matière sensible, les images qui s'y forment sont celles d'objets singuliers. La Sixième Méditation lui ajoute un second type de limite en invoquant l'exemple du chiliogone ${ }^{12}$ : l'incapacité de produire une image claire et distincte d'un tel objet. C'est là, certes, une limite épistémologique, dans la mesure où l'imagination est condamnée, comme les sens, à une connaissance confuse; mais cette limite répète en quelque sorte à l'intérieur de l'esprit ce qui, du point de vue du rapport entre les sens et le monde extérieur, constitue une limite physique. La conformation physique des sens fait qu'ils reçoivent du monde extérieur des impressions qui se traduisent en images confuses. L'entendement voulant produire sur l'imagination un chiliogone se voit renvoyer une image confuse impossible à distinguer d'un myriogone, de la même façon que l'impression sensible

10. Voir André CHARRAK, «L'imagination et la connaissance des choses matérielles selon Descartes et Leibniz», in Chantal JACQUET et Tamás PAVLOVITS (dir.), Les Facultés de l'âme à l'âge classique. Imagination, entendement, jugement, Paris, Publications de la Sorbonne, 2007, pp. 77-78.

11. «Mais ce qui est à remarquer, sa perception, ou bien l'action par laquelle on l'aperçoit, n'est point une vision, ni un attouchement, ni une imagination, et ne l'a jamais été, quoiqu'il le semblât ainsi auparavant, mais seulement une inspection de l'esprit, laquelle peut être imparfaite et confuse, comme elle était auparavant, ou bien claire et distincte, comme elle est à présent, selon que mon attention se porte plus ou moins aux choses qui sont en elle, et dont elle est composée » (Méditations, II, in Euvres de Descartes, op. cit., IXa, 24-25).

12. «Je remarque premièrement la différence qui est entre l'imagination et la pure intellection ou conception. Par exemple, lorsque j'imagine un triangle, je ne le conçois pas seulement comme une figure composée et comprise de trois lignes, mais outre cela je considère ces trois lignes comme présentes par la force et l'application intérieure de mon esprit; et c'est proprement ce que j'appelle imaginer. Que si je veux penser à un chiliogone, je conçois bien à la vérité que c'est une figure composée de mille côtés, aussi facilement que je conçois qu'un triangle est une figure composée de trois côtés seulement; mais je ne puis pas imaginer les mille côtés d'un chiliogone, comme je fais les trois d'un triangle, ni, pour ainsi dire, les regarder comme présents avec les yeux de mon esprit. Et quoique, suivant la coutume que j'ai de me servir toujours de mon imagination, lorsque je pense aux choses corporelles, il arrive qu'en concevant un chiliogone je me représente confusément quelque figure, toutefois il est très évident que cette figure n'est point un chiliogone, puisqu'elle ne diffère nullement de celle que je me représenterais, si je pensais à un myriogone, ou à quelque autre figure de beaucoup de côtés; et qu'elle ne sert en aucune façon à découvrir les propriétés qui font la différence du chiliogone d'avec les autres polygones » (Méditations, VI, in Euvres de Descartes, op. cit., IXa, 57-58). 
d'un chiliogone serait impossible à distinguer de celle d'un myriogone sans un décompte complet de leurs faces.

Ce parallélisme permet d'introduire la notion d'imagination passive. En effet, de même que l'entendement est, joint à la volonté, une force qui informe l'imagination et la détermine à produire des objets sensibles qui correspondent confusément à ce qui est pensé par lui, le monde extérieur est aussi constitué de forces qui agissent sur nos sens. Or, Descartes précise bien que les images qui résultent de cette action du monde extérieur sur les sens ne sont pas nécessairement ressemblantes :

Me proposant de traiter ici de la lumière, la première chose dont je veux vous avertir, est, qu'il peut y avoir de la différence entre le sentiment que nous en avons, c'est-à-dire l'idée qui s'en forme en notre imagination par l'entremise de nos yeux, et ce qui est dans les objets qui produit en nous ce sentiment, c'est-à-dire ce qui est dans la flamme ou dans le Soleil, qui s'appelle du nom de lumière. Car encore que chacun se persuade communément, que les idées que nous avons en notre pensée sont entièrement semblables aux objets dont elles procèdent, je ne vois point toutefois de raison, qui nous assure que cela soit; mais je remarque, au contraire, plusieurs expériences qui nous en doivent faire douter ${ }^{13}$.

Ainsi, la différence entre «l'idée qui se forme en notre imagination » des objets et les objets eux-mêmes rend compte du fait que des choses qui sont conçues par Dieu selon leur essence mathématique produisent en nous des images confuses. Comme ces images ne résultent pas d'une activité de notre entendement, ni de notre volonté, elles sont dites appartenir à l'imagination passive. Sous ce rapport, l'imagination n'est pas pouvoir de conception, mais de réception - d'une réception aussi limitée que l'est l'activité de conception. Les forces qui agitent le monde extérieur s'y voient converties en image par le jeu des esprits animaux qui traversent le système nerveux jusqu'au cerveau où ils impriment leur mouvement. On comprend par là les risques associés à l'imagination passive : le cours fortuit des esprits animaux peut faire qu'ils soient réintroduits dans des traces anciennes, produisant ainsi un effet de présence illusoire. Pourtant, même en ce contexte, l'imagination n'erre pas seule; les images qui y surviennent sont des effets de mouvements réels qui se produisent dans le corps.

Prise au sens général, donc, l'imagination n'est jamais qu'un pouvoir de figuration (ou de conception d'une figure), c'est-à-dire un matériau brut qui, sous l'impulsion de forces externes, peut prendre telle ou telle forme. La distinction entre ses formes passive et active indique simplement l'origine de ces forces. À titre de pouvoir de figuration, cependant, elle est la matière même où ces forces 
agissent, c'est-à-dire prennent leur réalité pour nous. Elle forme donc, jusqu'à un certain point, le tout de l'esprit, chaque pensée, chaque mouvement des esprits animaux y trouvant son lieu de manifestation. En régime cartésien, dès lors, l'imagination n'est susceptible ni de fausseté ni de vérité: les images qui s'y trouvent sont toujours le signe d'un événement réel. À tout prendre, l'imagination définit un espace où l'institution de la nature qui unit les deux substances se réalise en une trame unique de perceptions.

\section{MALEBRANCHE. IMAGINATION, INGENIUM, CONTAGION}

Les diverses remarques de Descartes sur l'imagination peuvent être considérées comme des corollaires de ce qu'il met en place pour résoudre des problèmes d'ordres divers qui se sont présentés à lui dans son effort pour trouver un fondement solide à l'édifice des sciences. L'enjeu final de ce travail est, bien sûr, de mettre ces sciences au service d'une meilleure connaissance de l'être humain, en vue de lui permettre de mieux régler son activité. Dans la Recherche de la vérité, cet arrangement est en quelque sorte inversé: Malebranche s'y donne pour tâche de délivrer l'être humain des causes occasionnelles de l'erreur, c'est-à-dire de ce qui l'éloigne de Dieu, afin de le rétablir dans le chemin de la vérité. L'anthropologie philosophique constitue donc ici le premier pas ; la méthode se révèle à la fin, comme un accomplissement du travail négatif qui a servi à neutraliser les causes d'erreur.

Cette manière de procéder permet à Malebranche de faire fond sur un héritage cartésien tout en en dégageant une version plus systématique là où il juge cet héritage lacunaire. Évidemment, cette systématisation ne va pas sans provoquer des déplacements d'accents: ainsi, après avoir rappelé la division entre imagination active et imagination passive ${ }^{14}$ dans des termes qui semblent directement empruntés au texte cartésien, Malebranche développe avec minutie tous les mécanismes de la seconde, mettant en place un cadre théorique qui servira, un peu plus loin dans la Recherche, à l'analyse des passions ${ }^{15}$. Ce cadre théo-

14. De la recherche de la vérité (texte établi par G. Rodis-Lewis), livre II, $1^{\text {re }}$ partie, chap. I, § 2, in Euvres complètes (sous la direction de A. Robinet), Paris, Vrin, 1962, vol. I, pp. 193-194. Désormais : $R V$, [ $\mathrm{n}^{\mathrm{o}}$ de livre], [ $\mathrm{n}^{\circ}$ de partie], [no de chapitre], [n $\mathrm{n}^{\circ}$ d'alinéa], $O C,\left[\mathrm{n}^{\circ}\right.$ de volume], [no de page].

15. Les passions sont abordées au livre V de la Recherche. Ainsi, l'imagination n'est pas, comme chez Descartes, une question traitée dans le cadre d'une théorie des passions, c'est-à-dire de l'âme en tant qu'elle est affectée par le corps. Ou plutôt: le terme de passion est réservé par Malebranche à ce qui relève de ce que l'on pourrait appeler l'affectivité ou les émotions de l'âme. Dès lors, on voit tout un ordre de phénomènes cognitifs être arrachés aux «passions » pour être repensés sous la catégorie d'imagination. En ce sens, imagination et passion sont comme des espèces distinctes de passivité. 
rique aura un mérite particulier: celui de fournir une explication qui assure une continuité sans faille entre les mécanismes physiologiques associés à l'imagination passive, c'est-à-dire aux causes physiques de la formation de représentations sensibles d'objets qui n'ont pas d'existence matérielle, et la transmission de ces représentations d'un esprit à l'autre. C'est là un des enjeux cruciaux d'un livre consacré aux causes d'erreur: il faut montrer qu'elles découlent de ce qui se passe en nous sans nous. Mais on ne saurait non plus en faire porter la responsabilité sur Dieu lui-même: aussi s'agit-il de les faire dépendre des lois du monde matériel, lesquelles répondent au principe de simplicité des voies. Autrement dit, l'imagination passive doit, dans un premier temps, voir tous ses effets reconduits à une cause générale, laquelle peut en même temps servir de principe de diversité :

Puis donc que l'imagination ne consiste que dans la force qu'a l'âme de se former des images des objets, en les imprimant pour ainsi dire dans les fibres de son cerveau, plus les vestiges des esprits animaux, qui sont les traits de ces images, seront grands et distincts, plus l'âme imaginera fortement et distinctement ces objets. [...] Ainsi la netteté des vestiges de l'imagination dépend de la force des esprits animaux, et de la constitution des fibres du cerveau ; et c'est la variété qui se trouve dans ces deux choses qui fait presque toute cette grande différence, que nous remarquons entre les esprits ${ }^{16}$.

Cette cause générale est donc inscrite dans le règne de la physiologie, qui, reposant sur des principes simples et universels, par le jeu des circonstances particulières auxquels les corps sont soumis, définit les modalités de l'individuation à l'intérieur d'une espèce. L'imagination est donc indéfectiblement attachée à ce qui, tant dans la constitution physique qui échoit à un individu que dans le cours des événements qui trame sa vie, fait qu'il devient ce qu'il est. Du sang qui coule dans les veines à l'épaisseur des fibres qui l'accueillent, des nourritures dont est tiré le chyle qui s'y mêle aux organes qui l'extraient, de l'usage du vin aux différents airs qui sont respirés, tout ce qui conditionne l'action des esprits animaux affecte la faculté d'imaginer de manière à lui donner l'aspect qu'elle a chez chaque être humain. En prenant ainsi à charge l'infinité de détails qui participent à la formation de l'individualité, l'imagination malebranchiste vient, en quelque sorte, prendre la place de l'ingenium conçu comme un don ou une pure innéité en la naturalisant. Sa loi est désormais celle d'un organisme qui évolue au rythme de ses interactions avec son environnement, elles-mêmes conditionnées par ses propres virtualités, et en conditionnant certaines ${ }^{17}$.

16. $R V$, II, I, II, § 3, OC, I, p. 194 .

17. Ainsi, toute la seconde partie du livre II sera consacrée à énumérer les traits propres à l'imagination des femmes, des personnes âgées, des gens d'étude, etc. 
Or, justement parce que les changements qui affectent les esprits sont occasionnés par de tels principes généraux, l'imagination d'individus distincts qui sont soumis à des déterminations similaires aura nécessairement aussi des traits communs. C'est là un point d'importance: si l'imagination est la faculté par excellence où se marque l'individualité, c'est aussi celle où apparaît l'appartenance de cette individualité à une sphère qui la dépasse, sphère constituée par l'ensemble des organismes qui interagissent dans un même environnement. L'ingenium se pense alors non seulement au niveau individuel, mais, aussi bien, au niveau d'un groupe chez qui un certain tour d'imagination se développe. À ce titre, la sphère d'appartenance peut avoir diverses extensions, selon le tour d'imagination que l'on considère. Les membres d'une famille, d'un rang social, d'une nation, etc. auront, à divers degrés, des penchants communs selon leur degré d'exposition aux divers facteurs qui agissent sur l'imagination (nourriture, coutumes, climat, etc.). Malebranche institue ainsi, au niveau physique strict, un ordre de causalité des phénomènes collectifs qui précède le jeu des institutions, et qui, dans une certaine mesure, lui donne ses conditions de possibilité, puisque celles-ci pourront alors être tenues comme des productions exprimant le génie de ceux qui les établissent. Cette naturalisation du politique, on s'en rend compte, disqualifie dans un même geste une approche contractualiste fondée sur la notion d'intérêt bien compris et une approche qui reposerait sur une «sociabilité naturelle », c'est-à-dire sur un penchant inné à aimer son prochain.

En ce qui a trait aux mécanismes physiologiques, Malebranche fait reposer son système sur la notion de sympathie. Le terme de sympathie confère son organicité au corps en faisant que tout changement dans le cours des esprits animaux a un effet sur la totalité du corps. Ainsi, elle est liée au processus par lequel, par exemple, les nerfs du visage, suite à l'action de quelque objet, transmettent du mouvement aux autres nerfs et, par suite, à la totalité du corps, préparant ainsi la réponse affective suscitée par l'objet ${ }^{18}$. Ainsi, la simple vue de quelque objet met en branle le nerf optique qui induit une réaction en chaîne sur la totalité des membres. Cette chaîne de réaction étant un effet naturel dû à la simplicité des voies de Dieu dans l'institution de la nature, elle se produit «par machine », sans ou contre l'action de la volonté, par l'ordre d'une providence qui a tout ordonné pour la conservation du corps ${ }^{19}$.

18. «La correspondance et la sympathie qui se trouve entre les nerfs du visage et quelques autres, qui répondent à d'autres endroits du corps, qu'on ne peut nommer, est encore bien plus remarquable ; et, ce qui fait cette grande sympathie, c'est comme dans les autres passions, que les petits nerfs qui vont au visage, ne sont encore que des branches de celui qui descend plus bas » $(R V, \mathrm{II}, \mathrm{I}, \mathrm{IV}, \S 3, O C$, I, p. 208).

19. Ibid., p. 209 : «Mais si l'on examine les raisons et la fin de toutes ces choses, on y trouvera tant d'ordre et de sagesse, qu'une attention un peu sérieuse sera capable de convaincre les personnes les plus attachées à Épicure et à Lucrèce, qu'il y a une providence qui régit le monde.» 
L'action sympathique explique la liaison qui existe entre les différentes parties du corps et assure la transmission causale du mouvement entre elles. Mais le modèle de cette action sympathique est transférable à d'autres liaisons matérielles. Malebranche entend ainsi montrer comment, suivant un principe de gradation, la sympathie qui assure l'unité du corps s'étend, selon certaines modalités spécifiques, à l'unité de la nature. L'institution divine assure que nous formons, à des degrés divers selon que notre conservation en dépend plus ou moins, un véritable corps avec ceux qui nous environnent ${ }^{20}$. L'existence d'une organicité de second ordre entre les membres d'un corps social donné introduit donc, cette fois par le biais de l'imagination, un régime de causalité qui se superpose à l'environnement physique, conditionnant avec une prégnance accrue le développement de sphères d'appartenance au sein desquelles l'individualité prend forme. Ce nouveau régime de causalité, fondé sur la naturalité des signes physiques associés aux différentes passions, rend possible la transmission des affections entre les individus. De cette nouvelle possibilité découlent la formation des langues et la communication des représentations qu'elles servent à signifier. De cette possibilité découle, donc, la formation d'une mémoire collective au sein de laquelle l'individualité est formée, mémoire qui attache cette individualité à d'autres qui la partagent.

Pour illustrer le principe de cette extension des relations sympathiques, Malebranche le décrit à partir de la relation la plus étroite que l'être humain entretienne avec un autre être, à savoir celui du fœtus avec sa mère. Ce premier degré de communication sert, en quelque sorte, de cas paradigmatique. Ainsi :

Quoique leur âme soit séparée de celle de leur mère, leur corps n'étant point détaché du sien, on doit penser qu'ils ont les mêmes sentiments et les mêmes passions, en un mot toutes les mêmes pensées qui s'excitent dans l'âme à l'occasion des mouvements qui se produisent dans le corps ${ }^{21}$.

Le fœtus ne fait qu'un avec le corps de sa mère. Dès lors, quoique leurs âmes soient distinctes, le mouvement des nerfs et des esprits animaux qui dispose le corps de la mère à l'occasion d'un objet et en produit l'image dans son cerveau se transmet au corps du fœutus et dispose son corps de la même façon. Il est donc

20. $R V$, II, I, vII, préambule, $O C$, I, pp. 233-234 : «Il est, ce me semble, assez évident que nous tenons à toutes choses, et que nous avons des rapports naturels à tout ce qui nous environne, lesquels nous sont très utiles pour la conservation et pour la commodité de la vie; mais ces rapports ne sont pas égaux. Nous tenons bien davantage à la France qu'à la Chine, au soleil qu'à quelque étoile, à notre propre maison qu'à celle de nos voisins. Il y a des liens invisibles qui nous attachent bien plus étroitement aux hommes qu'aux bêtes, à nos parents et à nos amis qu'à des étrangers, à ceux de qui nous dépendons pour la conservation de notre être, qu'à ceux de qui nous ne craignons et n'espérons rien. Ce qu'il y a principalement à remarquer dans cette union naturelle qui est entre nous et les autres hommes, c'est qu'elle est d'autant plus grande que nous avons besoin d'eux. »

21. $R V$, II, I, VII, § $1, O C, \mathrm{I}, \mathrm{p} .234$. 
ému avec sa mère, parce qu'il subit la même impression qu'elle. Or, comme le mouvement des esprits animaux est la cause occasionnelle de l'image qui se trace dans l'imagination, il faut admettre que l'impression subie par la mère et transmise à son fœtus doit produire une image similaire dans son esprit.

Ainsi les enfants voient ce que leurs mères voient, ils entendent les mêmes cris, ils reçoivent les mêmes impressions des objets; et ils sont agités des mêmes passions ${ }^{22}$.

L'institution de la nature qui fonde l'occasionnalisme de Malebranche assure la correspondance entre les traces dans le cerveau et les idées ${ }^{23}$ et entre les émotions et les mouvements des esprits animaux, de sorte qu'il n'est pas nécessaire d'être affecté par un objet pour en sentir la présence. Il suffit que les traces que cet objet susciterait le soient. Cet argument, Malebranche en tire les conséquences les plus radicales : la formation des monstres, par exemple, est attribuée à ces phénomènes de sympathie, sur la base du fait que ce qui frappe fortement l'imagination d'une mère sera littéralement reproduit sur le corps de l'enfant ${ }^{24}$, de même que la perpétuation des espèces, la transmission et la formation des inclinations et même de la concupiscence et du péché originel ${ }^{25}$.

Tout ce développement n'a, somme toute, pour raison d'être que de mettre en place les conditions nécessaires à l'explication des mécanismes de contagion qui définissent les modalités de fonctionnement des rapports imaginaires entre individus. Ainsi, ces ressorts de l'imagination sont immédiatement traités au chapitre des effets d'imitation qui portent à la compassion :

Il faut donc savoir que non seulement les esprits animaux se portent naturellement dans les parties de notre corps pour faire les mêmes actions et les mêmes mouvements que nous voyons faire aux autres, mais encore pour recevoir en quelque manière leurs blessures, et pour prendre part à leur misère ${ }^{26}$.

C'est là le sens de ce qui sera traité en détail dans la troisième partie du livre consacré à l'imagination, justement intitulé «De la communication contagieuse des imaginations fortes». On touche alors au point nodal de l'approche malebranchiste :

22. Id. [nous soulignons].

23. $R V$, II, I, v, $\S 1, O C$, I, p. 215 : «Toute l'alliance de l'esprit et du corps qui nous est connue consiste dans une correspondance naturelle et mutuelle des pensées de l'âme avec les traces du cerveau, et des émotions de l'âme avec les mouvements des esprits animaux. »

24. Malebranche cite l'exemple d'un nouveau-né naissant les membres rompus après que sa mère a vu un homme roué ( $R V$, II, I, vII, § 3, OC, I, p. 239).

25. $R V, \mathrm{II}, \mathrm{I}, \mathrm{VII}, \S 4,5$.

26. $R V$, II, I, VII, § 2, OC, I, p. 236. 
Les imaginations fortes sont extrêmement contagieuses : elles dominent sur celles qui sont faibles; elles leur donnent peu à peu leurs mêmes tours, et leur impriment leurs mêmes caractères. Ainsi ceux qui ont l'imagination forte et vigoureuse étant tout à fait déraisonnables, il y a très peu de causes plus générales des erreurs des hommes, que cette communication dangereuse de l'imagination ${ }^{27}$.

L'enjeu, en effet, est à ce stade de montrer comment la relation qui unit les êtres humains, si elle est moins intime que celle de la mère à son fœus, demeure fonctionnellement du même ordre, réglée par l'action sympathique. Le corps d'autrui affichant les signes d'une passion induit en moi une disposition similaire à la sienne, laquelle me dispose à la compassion, c'est-à-dire à subir une même impression ${ }^{28}$. Ainsi, la contagion qui s'effectue au niveau du corps peut-elle produire une contagion au niveau des représentations.

Il convient de noter que la difficulté soulevée ici par Malebranche commande la réintroduction de la distinction entre imagination active et imagination passive :

Ce n'est pas un défaut que d'avoir une imagination propre pour imaginer fortement les choses, et recevoir des images très distinctes et très vives des objets les moins considérables; pourvu que l'âme demeure toujours la maîtresse de l'imagination, que ces images s'impriment par ses ordres, et qu'elles s'effacent quand il lui plaît: c'est au contraire l'origine de la finesse et de la force de l'esprit. Mais lorsque l'imagination domine sur l'âme, et que sans attendre les ordres de sa volonté, ces traces se forment par la disposition du cerveau, et par l'action des objets et des esprits, il est visible que c'est une très mauvaise qualité et une espèce de folie ${ }^{29}$.

La force de l'imagination, en effet, n'est pas ce qui pose problème d'entrée de jeu; en revanche, Malebranche précise que les imaginations fortes sont généralement associées à une sensibilité outrée qui s'oppose à une attitude rationnelle. Le terme joue donc d'une ambiguilté : les imaginations fortes peuvent être des imaginations actives, c'est-à-dire maîtrisées par la raison, mais c'est rarement le cas, puisque la force d'imagination suppose des fibres plus aptes à recevoir des traces profondes suite à l'action des esprits animaux.

Mais c'est surtout dans leur rapport à d'autres esprits que les imaginations fortes deviennent problématiques. Les personnes à imagination forte, en effet, tendent à ressentir plus vivement ce qu'elles imaginent : leur corps tout entier est

27. $R V$, II, III, I, préambule, $O C$, I, pp. 320-321 [nous soulignons].

28. $R V$, II, III, I, $\S 1, O C$, I, p. 321 : «Pour concevoir ce que c'est que cette contagion, et comment elle se transmet de l'un à l'autre, il faut savoir que les hommes ont besoin les uns des autres, et qu'ils sont faits pour composer ensemble plusieurs corps, dont toutes les parties aient entre elles une mutuelle correspondance. »

29. $R V$, II, III, I, § 4, OC, I, p. 324. 
ébranlé avec plus de puissance, exprimant ainsi avec plus de force l'idée qui occupe leur esprit. Or, une telle agitation du corps n'est pas sans effet sur son environnement. Machinalement, tous ceux qui en perçoivent les mouvements sont, par le jeu de l'action sympathique, gagnés par des mouvements similaires, si bien que leur imagination se remplit des idées qui correspondent aux traces provoquées par la circulation des esprits animaux associée à ces mouvements.

Ainsi l'air de leur visage, le ton de leur voix, et le tour de leurs paroles animant leurs expressions, préparent ceux qui les écoutent et qui les regardent, à se rendre attentifs, et à recevoir machinalement l'impression de l'image qui les agite ${ }^{30}$.

Une telle contagion est problématique en ce qu'elle dérive d'un effet de persuasion qui se produit «plutôt par l'air et par l'impression sensible, que par la force des raisons $\gg 31$.

Les imaginations fortes ont donc ceci de particulier qu'elles parviennent, indépendamment de la source de leurs propres représentations, à produire un effet de présence en agissant sur l'imagination passive de ceux qui les côtoient. L'image qui les agite est alors transmise machinalement à d'autres esprits : ils voient ce qu'elles voient. Dans ce contexte, on comprend les risques associés à de tels effets de contagion: une simple illusion dans une imagination forte deviendra rapidement une réalité pour d'autres esprits. Malebranche montrera alors comment les effets de cette «communication d'imagination»sont amplifiés dès qu'une relation d'autorité est impliquée: de là la transmission d'airs de famille des parents vers les enfants, de là les modes créées par l'adoption, par une classe sociale, des pratiques de la classe supérieure. De là, surtout, l'impression que laissent les auteurs qui, comme Tertullien, Sénèque ou Montaigne, persuadent sans raison ${ }^{32}$.

Associer ainsi ces auteurs à l'effet qu'ils produisent sur l'imagination suffit pour les ranger dans la classe des propagateurs de représentations fausses. C'est là, à toute fin pratique, ce qui permet à Malebranche de joindre à la critique de ces auteurs celle des superstitions : à la toute fin de la section consacrée aux imaginations fortes, en effet, la Recherche de la vérité se penche sur les «sorciers par imagination » et les loups-garous. Ce qui est en fait visé concerne ces «superstitions qui ne se détruisent pas facilement ${ }^{33}$. Ces superstitions appartenant au champ ouvert par la théorie de l'imagination, elles constituent, dans le domaine des idées, l'élément qui correspond aux effets physiques de la sympathie, assu-

30. $R V$, II, III, I, $\S 6, O C$, I, p. 329.

31. Ibid., pp. 328-329.

32. $R V$, II, III, III, § 1, OC, I, pp. $341 s q$.

33. $R V$, II, III, VI, $\S 1, O C, \mathrm{I}$, p. 371 . 
rant la perpétuation des dérèglements de l'imagination qui nous tiennent éloignés de la raison et, donc, de Dieu.

Quand les hommes nous parlent, ils gravent dans notre cerveau des traces pareilles à celles qu'ils ont. Lorsqu'ils en ont de profondes, ils nous parlent d'une manière qui nous en grave de profondes, car ils ne peuvent parler qu'ils ne nous rendent semblables à eux en quelque façon. Les enfants dans le sein de leurs mères ne voient que ce que voient leurs mères; et même lorsqu'ils sont venus au monde, ils imaginent peu de choses dont leurs parents n'en soient la cause, puisque les hommes, même les plus sages, se conduisent plutôt par l'imagination des autres, c'est-à-dire par l'opinion et par la coutume, que par les règles de la raison ${ }^{34}$.

La théorie de l'imagination se voit ainsi attribuer dans un même geste les phénomènes relatifs à l'hérédité et ceux qui concernent la transmission des idées, rassemblant ainsi sous un même chef la mémoire biologique et la mémoire collective qui définissent les «caractères des nations ». L'individualité se forme ainsi sur le fond d'un héritage imaginaire - c'est-à-dire résultant du travail de l'imagination et de ses effets de contagion. L'imagination, à ce titre, est facteur d'historicité.

\section{FONTENELLE. IMAGINATION, FABLE, INVENTION}

L'emphase mise par Malebranche sur les mécanismes liés à l'imagination passive conduit, on l'a vu, à l'explication du processus de formation de l'opinion et des mœurs, lesquelles appartiennent désormais à un ordre entièrement naturalisé. Il devient alors possible de faire la genèse de l'état de l'opinion, en montrant en quel lieu précis l'imagination opère un passage qui transforme le fait psychologique en une production sociale, passage qui suppose d'abord un principe général :

Les hommes, s'attachant à tout ce qui est extraordinaire, se font un plaisir bizarre de raconter ces histoires surprenantes et prodigieuses de la puissance et de la malice des sorciers à épouvanter les autres et à s'épouvanter eux-mêmes ${ }^{35}$.

À ce principe général doivent alors se joindre les circonstances précises qui introduisent de la contingence dans l'histoire imaginaire que l'être humain se donne :

Un pâtre dans sa bergerie raconte après souper à sa femme et à ses enfants les aventures du sabbat. Comme son imagination est modérément échauffée par les vapeurs du

34. Ibid., p. 373.

35. $R V$, II, III, VI, $\S 1, O C, \mathrm{I}$, p. 370. 
vin, et qu'il croit avoir assisté plusieurs fois à cette assemblée imaginaire, il ne manque pas d'en parler d'une manière forte et vive. Son éloquence naturelle jointe à la disposition où est toute sa famille, pour entendre parler d'un sujet si nouveau et si terrible, doit sans doute produire d'étranges traces dans des imaginations faibles, et il n'est pas naturellement possible qu'une femme et des enfants ne demeurent tout effrayés, pénétrés et convaincus de ce qu'ils lui entendent dire ${ }^{36}$.

À cette naturalisation du procès de formation de l'opinion, qui dépend de l'imagination, et donc, en dernière analyse, des lois du monde physique, il n'y a d'échappatoire que dans l'action d'une volonté éclairée agissant, pour ainsi dire, de l'extérieur pour maîtriser l'imagination et l'arracher, ainsi, à sa passivité. En ce sens, sans dualisme, et sans conscience de cette dualité qui nous définit, rien, dans les processus associés à l'imagination seule, ne pourrait jamais arracher l'être humain à sa propre dérive historique.

Faisant écho à cette naturalisation de l'historicité de l'existence humaine, Fontenelle écrira Sur l'histoire et De l'origine des fables ${ }^{37}$ pour dégager les principes de cette historicité. À première vue, ces principes conditionnent une analyse génétique du même type que celle vue à l'œuvre chez Malebranche (que Fontenelle a déjà lu à ce moment, comme en témoigne la rédaction antérieure des Doutes sur le système physique des causes occasionnelles) :

Naturellement, les pères content à leurs enfants ce qu'ils ont fait, ce qu'ils ont vu; et sans doute cela s'est pratiqué dans les premiers siècles du monde. Ces récits doivent porter la marque de ces temps-là. Comme l'ignorance y était parfaite, la plupart des choses étaient des prodiges. Ainsi, un père ne manquait pas d'en remplir les contes qu'il faisait à ses enfants.

Quand on dit quelque chose de surprenant, l'imagination s'échauffe sur son objet, l'agrandit encore, et est même portée à y ajouter ce qui manquerait pour le rendre tout à fait merveilleux [...].

Les premiers hommes ont donc vu bien des prodiges, parce qu'ils étaient fort ignorants $[\ldots]^{38}$.

36. Ibid., p. 371.

37. Ces deux textes sont probablement de la période 1690-1699, bien qu'il n'existe pas, à ce jour, de preuve matérielle concluante pour pouvoir les dater. Alain Niderst, éditeur des œuvres complètes chez Fayard, a proposé (Fontenelle, OC, III, p. 4) de lire Sur l'histoire comme une ébauche de L'Origine des fables, parce que ce dernier est complet, alors que le premier semble être resté inachevé. Pourtant, Sur l'histoire est théoriquement beaucoup plus audacieux que L'Origine des fables, ce qui, à notre sens, pourrait indiquer que leur ordre d'écriture est inverse (nous remercions Claudine Pouloin, qui dirige actuellement une édition critique des œuvres complètes de Fontenelle, de nous avoir aidé à formuler cette hypothèse - encore à étayer dans un autre contexte - lors d'une journée d'étude sur ce dernier ayant eu lieu à Rouen au printemps 2009). Nous nous référerons ici aux deux textes comme s'ils appartenaient à un même groupe d'écrits sur l'histoire.

38. Sur l'histoire, OC, III, pp. 169-170. 
En dépit de l'apparente similitude entre ce développement sur l'origine des fables et la genèse de la superstition chez Malebranche, Fontenelle adopte en fait un cadre théorique qui en change radicalement le sens. En effet, celui-ci associe la formation de ces histoires non à une opération se faisant "par machine», mais à un travail de la curiositas: «Les hommes, écrit-il, sont toujours curieux, toujours portés à rechercher la cause de ce qu'ils voient ${ }^{39}$.»

$\mathrm{Au}$ cœur, donc, de ce qui met en branle l'imagination, on trouve, en lieu et place d'une simple réponse passive à un stimulus physique, un effort pour se ressaisir des faits. C'est pourquoi les récits fabuleux peuvent être considérés comme relevant d'une même activité originaire d'interprétation du monde :

À ces récits fabuleux, qui ne contenaient que des faits, se sont joints des systèmes de philosophie aussi fabuleux; car il y a eu de la philosophie même dans ces siècles grossiers $[\ldots]$

Il faut prendre garde que ces idées que nous appelons les systèmes de ces temps-là, étaient toujours copiées d'après les choses les plus connues [...]

La philosophie de ce temps-là et celle de celui-ci roulent sur un même principe ; c'està-dire que dans l'une et dans l'autre on ne fait qu'expliquer les choses inconnues de la Nature, par celles que l'expérience nous met devant les yeux, et transporter à la Physique les idées qu'elle nous fournit ${ }^{40}$.

Fontenelle n'hésite pas alors à nommer «systèmes d'imagination » ${ }^{41}$ les fables qui procèdent de cette impulsion originaire pour dépasser ce qui est donné dans l'expérience. De cette explication de la fable, on peut dégager deux conséquences pour la théorie de l'imagination:

1) D'abord, il apparaît que si les fables constituent un effet de l'imagination, celle-ci n'est en aucun cas traversée par une division entre sa forme passive et sa forme active. La curiosité qui pousse l'imagination à produire des représentations de l'inconnu à partir du connu définit une activité spontanée du sujet. À ce titre, Fontenelle réinterprète la formation des fables dans le sens d'une production de l'imagination active, d'un pouvoir de conception qui, par un jeu de transfert, généralise ce qui lui a été donné par les sens. Cette réinterprétation, c'est évidemment l'appartenance de Fontenelle au néo-épicurisme qui en conditionne la possibilité $^{42}$, lequel fonde la découverte des principes sur une théorie empiriste de l'abstraction. C'est pourquoi l'imagination, ici, n'est pas responsable par ellemême du caractère fabuleux des récits que les peuples de l'Antiquité ont laissés

39. Ibid., p. 170.

40. Ibid., pp. 170-171.

41. Ibid., p. 171.

42. Sur cette question, voir Jean DAGEN, «Fontenelle et l'épicurisme», Revue d'histoire littéraire de la France, CIII, 2003, 2, pp. 397-414. 
derrière eux ${ }^{43}$. L'ignorance seule l'est. Qu'est-ce à dire ? Fontenelle substitue à l'idée suivant laquelle l'imagination active est une imagination maîtrisée par l'action d'une substance séparée l'idée d'une action de l'imagination dirigée par l'état des connaissances empiriques. Ainsi :

Nous avons reconnu par l'usage, et non pas deviné, ce que peuvent les poids, les ressorts, les leviers; nous ne faisons agir la nature que par des leviers, des poids, des ressorts. Ces pauvres sauvages qui ont les premiers habité le monde, ou ne connaissaient point ces choses-là, ou n'y avaient pas fait attention. Ils n'expliquaient donc les effets de la nature que par des choses plus grossières et plus palpables qu'ils connaissaient ${ }^{44}$.

L'imagination est alors conçue comme une véritable puissance cognitive qui, procédant par analogie, explique la formation des entités théoriques en les faisant dépendre des conditions empiriques à l'intérieur desquelles elle s'élabore. Si c'est par l'usage qu'on reconnaît ce que peuvent les leviers, c'est par transfert de cette connaissance qu'on imagine des leviers derrière les effets de la nature, et qu'on parvient à en deviner le système ${ }^{45}$.

2) On comprend alors que l'opposition entre imagination active et imagination passive, qui permettait, chez Malebranche, de faire reposer la différence épistémologique entre science et superstition sur une différence ontologique, est chez Fontenelle replacée dans une perspective moniste qui historicise cette différence. Si les fables sont la philosophie d'une époque antique, au sens où elles proviennent des mêmes ressorts que la philosophie «moderne», c'est qu'elles sont déjà liées au travail conjoint de la volonté et de l'entendement sur l'imagination, mais d'un entendement qui manque d'expérience ${ }^{46}$. Ainsi, la valeur de vérité des

43. Sur l'histoire, $O C$, III, p. 174 : «On attribue ordinairement l'origine des fables à l'imagination vive des Orientaux ; pour moi, je l'attribue à l'ignorance des hommes. Mettez un Peuple nouveau sous le pôle, ses premières histoires seront des fables. »

44. Ibid., p. 171.

45. Dans les Entretiens sur la pluralité des mondes, Fontenelle écrit : «Toute la Philosophie, lui dis-je, n'est fondée que sur deux choses, sur ce qu'on a l'esprit curieux et les yeux mauvais ; car si vous aviez les yeux meilleurs que vous ne les avez, vous verriez bien si les Étoiles sont des Soleils qui éclairent autant de Mondes, ou si elles n'en sont pas; et si d'un autre côté vous étiez moins curieuse, vous ne vous soucieriez pas de le savoir, ce qui reviendrait au même : mais on veut savoir plus que ce qu'on ne voit ; c'est là la difficulté. Encore si ce qu'on voit, on le voyait bien, ce serait toujours autant de connu; mais on le voit tout autrement qu'il n'est. Ainsi, les vrais Philosophes passent leur vie à ne point croire ce qu'ils voient, et à tâcher de deviner ce qu'ils ne voient point; et cette condition n'est pas, ce me semble, trop à envier» $(O C$, II, p. 20).

46. C'est là, on s'en souviendra, un motif fondamental de la Digression sur les Anciens et les Modernes, et qui autorise une comparaison entre l'histoire de l'humanité et la vie d'un seul homme : «Ainsi cet homme qui a vécu depuis le commencement du monde jusqu'à présent, a eu son enfance, où il ne s'est occupé que des besoins les plus pressants de la vie; sa jeunesse, où il a assez bien réussi aux choses d'imagination, telles que la Poésie et l'Éloquence, et où même il a commencé à raisonner, 
fables n'est plus susceptible de détermination a priori à partir d'une considération sur leur provenance: vrais ou faux, les systèmes d'imagination qui apparaissent sur le terrain de l'histoire résultent des mêmes processus : ce sont des productions de l'esprit exprimant un état de connaissance que seule l'histoire peut inscrire sous le titre de fiction:

On nous a si fort accoutumés pendant notre enfance aux Fables des Grecs, que quand nous sommes en état de raisonner, nous ne nous avisons plus de les trouver aussi étonnantes qu'elles le sont. Mais si l'on vient à se défaire les yeux de l'habitude, il ne se peut qu'on ne soit épouvanté de voir toute l'ancienne Histoire d'un Peuple, qui n'est qu'un amas de chimères, de rêveries et d'absurdités. Serait-il possible qu'on eût donné tout cela pour vrai ? À quel dessein nous l'aurait-on donné pour faux ? Quel aurait été cet amour des hommes pour des faussetés manifestes et ridicules, et pourquoi ne durerait-il plus? Car les Fables des Grecs n'étaient pas comme nos Romans qu'on nous donne pour ce qu'ils sont, et non pas pour des Histoires; il n'y a point d'autres Histoires anciennes que les Fables ${ }^{47}$.

En ce sens, la nécessité dont elles procèdent n'est plus attachée à une passivité de l'âme qui pourrait faire l'objet d'un renversement par un acte de la volonté ; elle recouvre le prolongement des forces physiques dans l'esprit et l'institution du régime de causalité qui lui est associé, à savoir celui de l'historicité. Par là, Fontenelle permute, en quelque sorte, les rapports de dépendance qui instruisent les mécanismes de l'imagination. Celle-ci, en effet, est tôt arrachée à ses déterminations physiques par l'art et la culture, c'est-à-dire non par un effet de contagion, mais par celui du commerce ${ }^{48}$. Ainsi, s'il y a transmission d'une mémoire collective qui conditionne le tour d'imagination caractéristique d'un peuple, et les mœurs qui en dépendent ${ }^{49}$, celle-ci n'est pas le fait des lois du monde

mais avec moins de solidité que de feu. Il est maintenant dans l'âge de virilité, où il raisonne avec plus de force, et avec plus de lumière que jamais » $(O C$, II, p. 426).

47. De l'origine des fables, OC, III, p. 187.

48. Digression sur les Anciens et les Modernes : «Il est toujours sûr que par l'enchaînement et la dépendance réciproque qui est entre toutes les parties du monde matériel, les différences de climats qui se font sentir dans les plantes doivent s'étendre jusqu'aux cerveaux, et y faire quelque effet. Cet effet cependant est moins grand et moins sensible, parce que l'art et la culture peuvent beaucoup plus sur les cerveaux que sur la terre, qui est d'une matière plus dure et plus intraitable. Ainsi les pensées d'un pays se transportent plus aisément dans un autre que ses plantes, et nous n'aurions pas tant de peine à prendre dans nos Ouvrages le génie Italien, qu'à élever des orangers. Il me semble qu'on assure ordinairement qu'il y a plus de diversité entre les esprits qu'entre les visages. Je n'en suis pas bien sûr. Les visages, à force de se regarder les uns les autres, ne prennent point de ressemblances nouvelles; mais les esprits en prennent par le commerce qu'ils ont ensemble. Ainsi, les esprits, qui naturellement différeraient autant que les visages, viennent à ne différer plus tant» $(O C$, II, pp. 414415 - nous soulignons).

49. Sur l'histoire (OC, III, p. 183) : «Il y a une troisième chose qui résulte et des opinions de l'esprit et des passions du cœur; ce sont les mœurs des hommes, leurs coutumes, leurs différents usages.» Voir aussi: Vie de Corneille avec l'Histoire du Théâtre français jusqu'à lui et des 
physique, mais d'un échange d'idées et de ses conditions institutionnelles d'exercice. La philosophie d'un peuple, donc, loin d'exprimer son climat ou sa nourriture et de se transmettre par la sympathie physique qui lie ses membres, exprime plutôt l'état de ses connaissances empiriques et le réseau d'institutions qui sert leur distribution et leur transmission. C'est pourquoi «chez la plupart des Peuples, les Fables se tournèrent en Religion ${ }^{50}$ :

Les erreurs une fois établies parmi les hommes, ont coutume de jeter des racines bien profondes, et de s'accrocher à différentes choses qui les soutiennent ${ }^{51}$.

Ces deux traits de la pensée fontenellienne ${ }^{52}$ permettent donc une requalification complète de l'imagination: en contexte moniste, au lieu de voir son pouvoir réduit à celui d'instance sensible l'empêchant d'accéder au rang de faculté proprement cognitive, elle devient une extension de la sensibilité assurant l'extension du connu au-delà de l'expérience. Cette approche définit l'imaginable même comme la limite de l'intelligible: ainsi, la défense par Fontenelle de la théorie des tourbillons cartésiens contre le newtonianisme entrant en France s'autorise, justement, du fait que l'attraction est jugée inconcevable suite à la réduction des corps à leurs propriétés géométriques ${ }^{53}$. Or, comme on l'a vu, l'imaginable même se pense historiquement, c'est-à-dire dépend des faits qui ont fait l'objet d'une expérience.

Ces considérations affectent évidemment le statut épistémologique des productions de l'imagination. Ce que Fontenelle met en place, c'est une épistémologie qui s'organise autour des notions de vraisemblance et de systématicité ${ }^{54}$ :

Réflexions sur la Poésie, OC, III, p. 41 : «Les siècles diffèrent entre eux comme les hommes; ils ont chacun leur tour d'imagination qui leur est propre. »

50. De l'origine des fables, OC, III, p. 199.

51. Id. On a là, en bref, une explication de ce qui est longuement exploré dans l'Histoire des oracles, qui s'attache à montrer comment les dogmes religieux sont, le plus souvent, le fait d'une classe de la population qui abuse de son autorité pour entretenir une philosophie dont elle est la dépositaire, de manière à s'arroger une position sociale supérieure. A contrario, on peut lire, dans divers mémoires écrits pour l'Académie des sciences par Fontenelle, et notamment les Éloges des savants, plusieurs apologies de la liberté de parole qui règne à l'Académie, laquelle est garante du progrès des sciences et de la promotion sociale de ceux qui y participent sur la seule base du mérite.

52. Ces deux traits que nous avons identifiés renvoient à un passage de L'Origine des fables (OC, III, pp. 195-196) : «Outre tous ces principes particuliers de la naissance des Fables, il y en a deux autres plus généraux qui les ont extrêmement favorisées. Le premier est le droit que l'on a d'inventer des choses pareilles à celles qui sont reçues, ou de les pousser plus loin par des conséquences [...] Le second principe qui sert beaucoup à nos erreurs, est le respect aveugle de l'antiquité. Nos pères l'ont cru; prétendrions-nous être plus sages qu'eux ?» Ainsi, activité productrice de l'imagination et force d'inertie que fait peser sur l'esprit ce qui est transmis forment les deux composantes les plus générales de la théorie de l'imagination de Fontenelle.

53. Éloge de M. Newton, OC, VII, pp. 118 sq.; Théorie des tourbillons cartésiens, OC, VII, pp. 453 sq.

54. Sur l'histoire, $O C$, III, p. 176 : «Le Philosophe a devant lui un certain nombre d'effets de la 
l'expérience permettant de confirmer et d'infirmer les différents systèmes qui se sont succédé dans l'histoire, le cercle du vraisemblable se rétrécit. Nous profitons des erreurs du passé dans la mesure où elles nous renseignent sur ce qu'on peut désormais considérer comme faux. Les systèmes, alors, se perfectionnent, tout en demeurant frappés d'incomplétude. C'est là ce qui fait du progrès une étendue virtuellement infinie ${ }^{55}$.

Si l'imagination est facteur d'historicité chez Fontenelle, c'est donc en un sens différent de chez Malebranche : chez ce dernier, l'histoire est le résultat des lois du monde matériel, et les déterminations qui en découlent, même si elles viennent de la simplicité des voies choisies par Dieu, font de l'historicité de l'existence humaine un régime d'existence irrationnel; chez Fontenelle, au contraire, l'historicité est un régime de causalité dont émerge un ordre culturel qui arrache l'être humain aux lois du monde matériel et le fait entrer dans un régime d'existence à la rationalité croissante. Ainsi, pour l'un, l'histoire est le lieu d'une perte de maîtrise de soi; pour l'autre, elle est le lieu de sa conquête. En ce qui a trait à l'imagination en tant que telle, elle constitue chez les deux penseurs l'opérateur fondamental de ce qui se joue sur la scène de l'histoire.

\section{CONCLUSION}

Ces dernières remarques font voir à quel point le cartésianisme recelait en son sein des extensions possibles très diverses. Si la pensée de Descartes peut, en quelque sorte, être considérée comme un espace d'équilibre entre deux polarités, celles de Malebranche et de Fontenelle peuvent être prises comme des accentuations de ces polarités. Le concept d'imagination constitue, nous semble-t-il, un révélateur des modalités de réception et de développement de cette pensée. Par les tournures particulières qu'il prend chez Malebranche et Fontenelle, il acquiert une double configuration, qui circonscrit les lieux théoriques au sein desquels il jouera un rôle important au XVIII ${ }^{\mathrm{e}}$ siècle.

L'un des plus importants de ces lieux théoriques concerne évidemment la question de l'historicité. Ce que la théorie de l'imagination qui se met en place à la fin du XVII ${ }^{\mathrm{e}}$ siècle en France apporte de plus fondamental, c'est une confi-

nature et d'expériences; il faut qu'il en devine des causes vraisemblables, et que de ce qu'il voyait, et de ce qu'il devine, il en compose un tout bien lié; voilà le système. »

55. Digression sur les Anciens et les Modernes, OC, II, p. 426 : «Je suis obligé d'avouer que cet homme-là [celui formé de l'ensemble des hommes] n'aura point de vieillesse; il sera toujours également capable des choses auxquelles sa jeunesse était propre, et il le sera toujours de plus en plus de celles qui conviennent à l'âge de virilité ; c'est-à-dire, pour quitter l'allégorie, que les hommes ne dégénéreront jamais, et que les vues saines de tous les bons esprits qui se succéderont, s'ajouteront toujours les unes aux autres. » 
guration conceptuelle qui explicite en la naturalisant la dynamique qui préside à la formation de la «philosophie» d'un siècle, d'une nation, d'une fraction de la société. Parce que l'imagination est à la fois liée à l'expérience sensible, à la mémoire, à l'invention et à la communication, elle devient par le fait même le terrain sur lequel s'établit un rapport entre l'expérience concrète que nous faisons du monde et la manière dont cette expérience est interprétée. En elle se produisent les effets divers qu'on peut attendre de la concaténation des «causes physiques» et des «causes morales» qui président à la formation de l'opinion privée, à son institution dans la sphère publique, et à l'effet en retour de cette institution. En ce sens, c'est toute la construction du concept moderne de culture qui s'y joue.

Mitia RiouX-Beaulne

Université d'Ottawa 